CORRECTION

https://doi.org/10.1038/s41586-018-0412-8

\title{
Publisher Correction: Controlling an organic synthesis robot with machine learning to search for new reactivity
}

Jarosław M. Granda, Liva Donina, Vincenza Dragone,

De-Liang Long \& Leroy Cronin

Correction to: Nature https://doi.org/10.1038/s41586-018-0307-8, published online 18 July 2018.

In structure 19 of Fig. $5 \mathrm{a}$ of this Letter, the mixed double bond configuration $(E / Z)$ was represented by a crossed double bond, rather than a wavy bond. Figure 5 has been corrected online. 